\title{
Diferenciación Social en patrones de consumo de exposiciones de arte, teatro, danza, recitales en vivo y circo en la sociedad chilena
}

\author{
JOSÉ IGNACIO NAZIF'
}

\section{Resumen}

Este estudio analiza la estratificación social del consumo de exposiciones de arte, teatro, danza, recitales en vivo y circo en la sociedad chilena. De todas las variables sociológicas que predicen el consumo cultural, se destacan nivel educacional, influencia de la familia y el Índice realización individual de actividades artísticas. Estos resultados cuestionan, por una parte, los postulados que Bourdieu ha realizado en esta área, y por otra, reflejan una tensión de los procesos de modernización que esta sociedad tiene, puesto que el consumo cultural transita entre estructuras sociales tradicionales y modernas. Finalmente es posible detectar un grupo "omnívoro" (personas que consumen bienes culturales de distinta índole) duro, el cual realiza un consumo transversal de todos lo bienes culturales analizados. Los datos de este estudio provienen de la encuesta nacional de consumo cultural 2005 realizada por el Consejo Nacional de la Cultura y la Artes.

PALABRAS CLAVE: Estratificación social, consumo cultural, Bourdieu

\begin{abstract}
This study analyzes the social stratification of art exposition, theater, dance, live musical shows and circus consumption within Chilean society. From every sociological variable which predict cultural consumption only educational level, family's influence and the individual artistic activity performance index have a significant impact. These results, on the one hand, question Bourdieu's cultural consumption theory, and on the other, reflect a tension of the modernization process which this society has, since cultural consumption travels between traditional and modern social structures. Lastly, it is possible to detect a hard omnivore group (people who consume cultural goods of different kind), which transversally consumes every single cultural good analyzed. Data of this study was taken form the national cultural consumption survey carried out by the Culture and Arts National Commission.
\end{abstract}

KEY WORDS: Social stratification, cultural consumption, Bourdieu

José Ignacio Nazif, Sociólogo, Universidad de Chile. Master of Arts in Sociology, McGill University. Quisiera agradecer a Alberto Mayol y Pablo Morris (sociólogos políticos de pura cepa) por sus importantes colaboraciones en versiones anteriores de este artículo. La responsabilidad por el análisis e interpretación realizados es exclusivamente mía. 
Revista de Sociolocía 21 / 2007 Chile Hoy

Facultad de Ciencias Sociales - Universidad de Chile

\section{Introducción}

Dentro de los estudios sociológicos de estratificación social, los análisis de consumo o gusto cultural han sido parte importante de un debate extenso. El objetivo principal de este trabajo es examinar en la sociedad chilena la estratificación social del consumo de exposiciones de arte, obras de teatro, espectáculos de danza, recitales en vivo y espectáculos circenses. Dicho trabajo comienza con una breve discusión teórica de cuatro posiciones sociológicas, las cuales han estado presentes en la literatura especializada. A éstas las clasificaremos como: i) Distinguidos; ii) Cuasiposmodernos; iii) Omnívoros-unívoros; y iv) El eslabón perdido (la familia). En segundo término son presentados los resultados del análisis estadístico efectuado. Ellos ilustran en primer lugar qué tipo de consumo cultural hay en la sociedad chilena, pero sobretodo ayudan a entender empíricamente qué patrones sociológicos son correlacionados con la asistencia a exposiciones de arte, obras de teatro, espectáculos de danza, recitales en vivo y circo. Cabe señalar que esto es de suma importancia por cuanto al conocer cómo está configurado socialmente el consumo cultural es posible destacar parte importante del proceso de modernización que esta sociedad vive. Finalmente, este trabajo cerrará con una breve reflexión respecto a por qué, para la sociedad chilena, sólo algunas de las propuestas teóricas son adecuadas para entender el fenómeno en cuestión.

\section{Explicando sociológicamente el consumo cultural}

Se destaca en la literatura tres posiciones sociológicas (distinguidos; cuasiposmodernos; y omnívoros-unívoros). Cada una de éstas presenta diferentes respuestas a las preguntas por qué y cómo están estratificados el consumo y el gusto cultural en las sociedades contemporáneas. No obstante lo anterior, cabe explorar una cuarta posición, siendo ésta la influencia que ejercen los miembros mayores de las familias sobre sus descendientes. La incorporación de esta última alternativa obedece a dos elementos; primero, ésta no ha sido explícitamente introducida en estos tipos de análisis, y en segundo lugar, para el caso de la sociedad chilena resulta de especial interés por cuanto la familia mantiene su preponderancia en la socialización de los individuos. En lo que viene cada una de estos enfoques será analizado.

i) Los distinguidos

La centralidad de esta explicación está basada en la constatación de que existe un solapamiento muy fuerte de la estratificación social con la estratificación cultural, es decir, la condición de clase social puede revelar, con altas probabilidades, el consumo de un bien cultural específico. ${ }^{2}$ Así, los cánones artísticos de alto nivel están ligados al consumo realizado por la elite, mientras que el consumo de cultura de masas a los estratos sociales bajos. Bourdieu ha sido por antonomasia uno de los autores que llevó

Respecto de algunos estudios relacionados con dicha teoría véase: Gans, H.J (1999: 89-159); Mukerji y Schudson (1999: 1-54) y Savage et al. (2005). 
Diferenciación Social en Patrones de Consumo de Exposiciones de arte, teatro, danZA, ReCitales en Vivo y CIRCo en la Sociedad Chilena / José IGnacio Nazif

a cabo dicho programa de investigación con mucho éxito, por cuanto desarrolló tanto una metodología ad-hoc para entender la complejidad del fenómeno, como también porque los resultados encontrados en una de sus principales obras, La Distinction, avalaron empíricamente, para el caso de la sociedad francesa, el correlato teórico

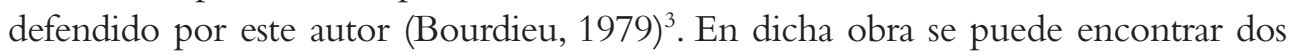
mecanismos sociales que explican la formación del consumo y gusto cultural. En primer lugar, se destaca aquél donde las diferencias culturales se refuerzan por elementos subjetivos que los actores utilizan para poder autodiferenciarse con miembros de otras clases y también con fracciones de la misma clase.Aun cuando algunos actores arguyan pureza o placer desinteresado en el gusto o consumo del artefacto cultural en cuestión, Bourdieu señala que dicha expresión sólo busca esconder la noción de superioridad y de distinción con el otro. Según este autor este es un mecanismo que permite reificar la reproducción de la distinción de clase. En segundo lugar se encuentra el amor fati o le choix du nécessaire, aquí simplemente el actor está impedido de poder elegir por cuanto está constreñido por diversos recursos a probar otros gustos culturales o bien a consumir otros artefactos (Bourdieu, 1979). Este último mecanismo está asociado a la clase trabajadora o a representantes de clases bajas. ${ }^{4}$ En resumen Bourdieu sugiere que parte de la vida social de la elite se produce pour-autri, mientras que para la clase trabajadora en-soi. ${ }^{5}$

Finalmente uno de los elementos que sobresale en la propuesta teórica de Bourdieu es la diferencia que establece con Weber. Contrario al autor alemán, éste señala que la distinción analítica entre clase y status no es procedente por cuanto esta última categoría es la referencia simbólica de la estructura de clase, es decir, no sería apropiado establecer una relación contingente entre ambas dimensiones. Es este campo simbólico el que provee a algunos actores la capacidad de poder distinguirse con respecto a los miembros de las otras clases. Para Bourdieu es importante destacar como una acción simbólicamente violenta la que la elite ejerce para diferenciarse, por cuanto dicho grupo arguye tener el monopolio del canon (Bourdieu, 1979).

ii) Cuasiposmodernos

Las proposiciones teóricas cuasiposmodernas se pueden ubicar en una posición virtualmente opuesta a la de los “distinguidos”. Éstas señalan que la distribución del consumo cultural sobrepasan los patrones de estratificación social. Es decir, el tipo de consumo cultural de las personas no está anclado a la herencia de su clase de origen, sino más bien por proyectos de vida personales, los cuales están marcados por la posibilidad

\footnotetext{
Es necesario señalar que la aplicación de la teoría de Bourdieu en este trabajo se hace con herramientas metodológicas completamente diferentes a las que este autor desarrolló para el estudio del campo cultural. Dos trabajos de similares características pueden ser vistos en Fernández (2006) y García-Albarido (2005).

Este último mecanismo es el descrito por Elster (1983) como sour grapes.

Respecto de la ambigüedad e inconsistencia del funcionamiento de estos planteamientos y en especial de los mecanismos asociados al funcionamiento de la estructura de clase en la obra de Bourdieu véase Elster, Jon (1981) y van den Berg, Axel (1998).
} 
de realizar su propia reconstrucción. Una de las explicaciones propuestas por Giddens para entender las tensiones de la construcción del self puede ser clasificada dentro de estas propuestas teóricas. Al respecto dicho autor señala que la construcción del self en las sociedades modernas pasa por una tensión constante la cual deviene en diversas materializaciones de la constitución del self. En consecuencia los patrones culturales se pueden enmarcar en dilemas provocados por elecciones personales provenientes de experiencias de unificación y fragmentación, de impotencia y apropiación, autoridad e incertidumbre, y experiencias personificadas y mercantilizadas (Giddens, 1991). Un segundo autor asociado a este tipo de propuestas téoricas es Beck. Dicho sociólogo señala que, para el caso de algunas sociedades donde han sido promovidos modelos de estados de bienestar, las condiciones de clases y status ya no explican los patrones de consumo cultural o la formación de biografias personales. Las reformas que han promovido cambios en la movilidad social, la extensión de la educación en la población, la ampliación del tiempo libre por contraposición a la disminución de las jornadas laborales, la mayor participación de la mujer en el mercado laboral remunerado y la extensión de la duración de la vida, han repercutido en una destradicionalización del funcionamiento de las clases sociales, y con ello se activa un fuerte proceso de individualización. ${ }^{6} \mathrm{Al}$ igual que Bourdieu, este autor también hace una referencia al trabajo de Weber. Sin embargo su crítica se ubica en una posición completamente contraria ya que Beck entiende que el ordenamiento biográfico de los sujetos supera la vigencia de las tradiciones y las subculturas estamentales. En resumen si antes había clases y status como dos entes ordenadores, en la actualidad, las características aglutinadoras se conforman a partir de la institucionalización de la individualización. Beck las reconoce a éstas como momentos biográficos o fases de la vida (Beck, 1992).

Los estudios, que refuerzan dichas teorías para el consumo y gusto cultural, entienden que el proceso de modernización de las sociedades, el cual se manifiesta en importantes cambios estructurales, va permitiendo que los sujetos tengan mayores posibilidades de ir ejerciendo sus elecciones. En otras palabras, en dicho proceso las fuentes tradicionales de socialización tales como la familia, la escuela y la clase van perdiendo su fuerza por una parte, y por otra, los sujetos refuerzan sus posibilidades de ampliar sus identidades debido a la vasta cantidad de productos (culturales) que la sociedad de consumo ofrece. Cada uno de estos bienes en manos de los actores va tomando un significado especial en conformidad a las biografias personales e influyendo en los proyectos de vidas. Rasgos de esta teoría han sido operacionalizados a través de la incorporación de variables como edad, género o la herencia étnica de los individuos (Lipovetsky, G. et E. Roux; Boden et al., 2004; Livingston, 2004; Biggs et al., 2006; Higgs et al., 2006).

\footnotetext{
Lo anterior no significa, señala Beck (1992), que en dicho tipo de sociedades no persistan relaciones de desigualdad, sino más bien que hay un "efecto ascensor donde la "sociedad de clases" es llevada más arriba".
} 
Diferenciación Social en Patrones de Consumo de Exposiciones de arte, teatro,

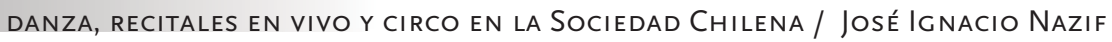

iii) Omnívoros-unívoros

Esta tercera explicación es una síntesis de las dos posiciones anteriores. Por una parte, entiende que el solapamiento entre estratificación social y estratificación cultural es más endeble ya que el consumo cultural no sigue en forma estricta la distinción elite-clases bajas. Según Peterson y Simkus (1992) es incorrecto entender el dominio de gusto cultural como una pequeña columna donde los gusto de la elite y las clases populares están en la parte superior e inferior respectivamente, sino más bien como una pirámide invertida. Es decir, dichos autores señalan que el consumo cultural de la elite ya no está exclusivamente asociado a formas distinguidas de arte, puesto que éste también se extiende a algunas otras formas que han estado relacionadas con la cultura de masas. Es decir, la elite consume más formas de expresión cultural, y por otra parte los miembros de las clases bajas mantienen el consumo de expresiones culturales populares. Conforme a esta línea de trabajo teórico Bryson, van Eijck, y Chan y Goldthorpe, han desarrollado una agenda de investigación en el cual se encuentra análisis sobre consumo de música, teatro, danza, cine, televisión, entre otros (Bryson 1996; van Eijck 2001; y Chan y Goldthorpe 2007a, 2007b, 2006, 2005, y 2004).

Chan y Goldthorpe conceden que esta versión puede ser sin embargo fuente de una doble interpretación la cual no supera la contradicción de las versiones distinguidas y cuasiposmodernas. En primer lugar los omnívoros pueden ser entendidos como personas tolerantes (debido principalmente a sus altos niveles educacionales, o bien porque han experimentado movilidad social) quienes tienen una especial apertura a diversas expresiones culturales debido al deseo de experimentar con diversas alternativas culturales o bien porque han sido socializados en dicha manera. Evidentemente, estos autores señalan, bajo esta interpretación hay una afinidad obvia con las interpretaciones cuasiposmodernas. Lo anterior porque las personas omnívoras estarían más vinculadas a procesos de autorealización personal que a establecer con dicho consumo una distinción de clase y poder en consecuencia diferenciarse en términos simbólicos.Y en segundo término, los omnívoros aun cuando expresan una nueva forma estética, la cual puede ser etiquetada como cosmopolita o incluyente, especialmente cuando son tenidas como referencias las elites tradicionales, puede también ser parte de una estrategia de distinción o superioridad al contraponerse a los estilos culturales de los unívoros (Bellavance,Valex y Ratté, 2004). Con lo anterior los omnívoros, estarían discriminando a través de dos formas, relacionarse irónicamente con las expresiones artísticas culturales de orden popular o bien rechazando aquellas que están mayormente vinculadas a status sociales más bajos. Esto último va en línea con la propuesta teórica de Bourdieu ya que el consumo cultural sería una forma que mantendría la frontera simbólica en términos de competencia y distinción (Chan y Goldthorpe, 2006, 2005 y 2004).

Finalmente, Chan y Goldthorpe, a diferencia de Bourdieu y Beck, entienden que la distinción weberiana de clase y status, tiene mucha relevancia mantenerla. Ello 
porque ambos aspectos explican diferenciadamente algunas conductas o actitudes de los sujetos, i.e. clase predice mejor las actitudes políticas ubicadas en el continuo 'izquierda-derecha', mientras que status sería un mejor predictor de las actitudes 'libertario-autoritario' (Chan y Goldthorpe, 2006). En coherencia a lo anterior, dichos autores demuestran que status tiene mayor fuerza explicativa que clase para entender la distribución del consumo cultural, sea este en el área de la música, consumo de diarios, o bien el teatro, danza y presencia en el cine (Chan y Goldthorpe, 2007a, 2007b, 2006, 2005).

iv) El eslabón perdido (la familia)

Considerar a la familia como una última alternativa para explicar el consumo de bienes culturales obedece a dos elementos sociales, uno de orden macro y otro asociado a los mecanismos sociales que estos grupos producen. En primer lugar, debido a que esta sociedad contiene estructuras sociales tradicionales que marcan fuertemente su propia dinámica, se puede señalar que Chile vive un proceso de modernización diferente al de sociedades desarrolladas. (Larraín, 2001 y 1997; Barozet, 2006). Tomando por ejemplo en consideración algunos indicadores señalados por Beck para identificar a las sociedades desarrolladas, i.e. la disminución de la jornada laboral, los altos niveles de movilidad social, o la incorporación sustantiva de las mujeres al mercado remunerado, se puede señalar que en Chile cada uno de estos factores presenta todavía rezagos muy significativos. Valenzuela y Herrera demuestran empíricamente que el tiempo de trabajo en Chile es superior a varios países europeos y también al caso norteamericano (Valenzuela y Herrera, 2006: 274). Con respecto a la movilidad social, según análisis efectuados por Contreras et al. y por Torche, se puede sugerir que el grupo establecido en la parte alta de la sociedad está prácticamente clausurado dado que la movilidad hacia éste es todavía muy baja (Contreras et al.,2004; Torche,2005). Como lo señala Larrañaga, a pesar de los desarrollos de las últimas décadas y cuando es tomado como punto de referencia el estándar internacional, el nivel de la participación laboral de la mujer sigue siendo bajo (Larrañaga, 2006:182 y 211). En conformidad a lo anterior, se puede sugerir que el consumo de bienes culturales no necesariamente se da en coherencia a los planteamientos cuasiposmodernos aquí revisados, sino que las exploraciones biográficas que podrían realizar los individuos en torno al consumo de estos bienes estarían sujetas todavía a posiciones estructurantes de índole más tradicional, en cuyo caso el rol de la familia puede entregar evidencia importante.

Con respecto a los mecanismos sociales, y tal como lo señala Tironi, Valenzuela y Scully la familia despliega al menos cuatro funciones: reproducción, protección, comunidad, y socialización. Interesa esta última como otra alternativa para explicar el consumo de bienes culturales, ya que alli "se aprenden las normas, valores y todos los aspectos propios de la vida social; se adquieren el lenguaje y las costumbres, los gustos y las preferencias, los hábitos de trabajo y de estudio, el respeto y valoración del 
Diferenciación Social en Patrones de Consumo de Exposiciones de arte, teatro, dANZA, ReCitAles en Vivo y CiRCo en la Sociedad Chilena / José IGnacio Nazif

otro" (Tironi, Valenzuela y Scully, 2006: 18) o como lo sintetiza Winch, la familia es "la principal puerta de acceso a la vida social. Es la que otorga una posición inicial a sus miembros, mediante la transmisión de valores y el status social de los padres" (Winch, 1963). Para el caso particular de la familia latinoamericana, Jelin observa que esta es una institución "mediadora entre la estructura social en un momento histórico dado y el futuro de esa estructura social” (Jelin, 1994:98). En Chile hay investigaciones que destacan precisamente el rol de la familia en el aprendizaje de los individuos en varios aspectos. Por una parte, Brunner y Elacqua, y Fontaine, señalan que el rendimiento escolar está asociado al tipo de familia en la cual se nace (Brunner y Elacqua, 2003; Fontaine, 2002). Por otra parte, Valenzuela muestra resultados similares cuando es analizada la prevención de conductas asociadas al consumo de cocaína, marihuana y alcohol. Dichos estudios sugieren que el principal mecanismo social es el involucramiento de los padres en el control de las actividades que los hijos realizan (Valenzuela, 2006). Si bien el control puede ser un mecanismo que dichos grupos realicen, hay otros mecanismos presentes que también podrían explicar el consumo de bienes culturales. Al respecto la imitación racional ${ }^{7}$ que realizan las personas se materializa en acciones de consumo de ciertos bienes culturales, por cuanto ellos entienden que las actividades anteriormente desarrolladas por miembros mayores de su núcleo familiar son acciones legítimas de seguir repitiendo.

\section{Metodología}

\section{i) Datos}

Los datos utilizados en este estudio provienen de la Encuesta de Consumo Cultural realizada por el Consejo Nacional de la Cultura y las Artes durante el año 2005. Dicha encuesta entrevistó en forma aleatoria a 4.603 personas utilizando entrevistas cara a cara. Para efectos de revisar las tesis aquí estudiadas se procedió a eliminar a las personas que estaban fuera del mercado laboral (dueñas de casa y estudiantes), menores de 25 años y mayores de 60 años. Lo anterior porque el tiempo libre de cada una de estas personas puede afectar significativamente el análisis. Particularmente para el caso de ambos grupos etáreos y tal como lo sugieren Chan y Goldthorpe, su exclusión obedece a que ellos tienen particulares modos de consumo que ameritan aproximaciones distintas (Chan y Goldthorpe, 2005:210). La primera muestra para hacer el análisis fue de 1.853 casos. Cabe señalar que para efectos de analizar el consumo de circo, se trabajó con una muestra de 1.349 casos, ello debido a que había un número mayor de casos perdidos.

\section{ii) Dimensiones}

A objeto de poder entender como está estratificado socialmente el consumo cultural, éste se operacionalizó en tres dimensiones:

Sobre la definición de imitación racional ver Hedström, Peter (1998). 
ReVista de Sociolocía 21 / 2007 ChILe Hoy

Facultad de Ciencias Sociales - Universidad de Chile

a) Dimensión Asistencia a exposiciones de arte: este consiste en saber si un sujeto entrevistado había asistido (0) o no (1) a una exposición de arte.

b) Dimensión Índice consumo de teatro, danza y recitales en vivo: este consiste en las respuestas que los sujetos dieron respecto a cuántas veces habían ido al teatro, danza y recitales en vivos tomados todos al mismo tiempo. Para el consumo de cada una de expresiones las alternativas eran: (1) 'nunca'; (2) 'ninguna' (3) '1 vez'; (4) '2 o 3 veces'; (5) ‘4 o 5 veces'; y (6) 'más de 6 veces'. Para obtener el resultado de este índice se promedió el puntaje asociado a cada una de las alternativas de las tres preguntas. El alfa de Cronbach de este índice es de 0,66 .

c) Dimensión Asistencia al circo: este consiste en saber cuántas veces un sujeto entrevistado había asistido espectáculos circenses. Las alternativas fueron: (1) 'nunca'; (2) 'ninguna' (3) '1 vez'; (4) '2 o 3 veces'; (5) 'más de 4 veces'.

Se decidió realizar esta división del consumo cultural en las tres dimensione señaladas más arriba por lo siguiente: en primer lugar, la categorización de las alternativas para la pregunta relacionada con el consumo de exposiciones de arte no permitía realizar un promedio con los puntajes asociados con las otras expresiones culturales aquí analizadas; segundo, la dimensión asistencia al circo fue analizada en forma separada dado que ésta en sí representa una expresión cultural completamente distinta a las formas "elitistas" de arte. Sin embargo, para la construcción del índice de consumo cultural dicho ítem fue introducido en una primera circunstancia puesto que de esa manera se podía revisar con mayor precisión la tesis "omnívoros-unívoros".

\section{Tabla 1. Estadísticas Descriptivas De LAS Dimensiones}

\begin{tabular}{|c|c|c|c|c|}
\hline Dimensiones & Alternativas & $\%$ & Media & $\begin{array}{l}\text { Desviación } \\
\text { Standard }\end{array}$ \\
\hline \multirow{2}{*}{$\begin{array}{l}\text { a) Asistencia a exposiciones } \\
\text { de arte }\end{array}$} & (0) Asistió & 25,7 & & \\
\hline & (1) No asistió & 74,3 & & \\
\hline $\begin{array}{l}\text { b) Índice consumo de teatro, } \\
\text { danza y recitales en vivo }\end{array}$ & & & 2,228 &, 778 \\
\hline \multirow[t]{5}{*}{ c) Asistencia al circo $^{1}$} & (1) Nunca & 2,4 & & \\
\hline & (2) Ninguna & 74,6 & & \\
\hline & (3) $1 \mathrm{vez}$ & 14,2 & & \\
\hline & (4) 2 o 3 veces & 6,8 & & \\
\hline & (5) Más de 4 veces & 2,0 & & \\
\hline
\end{tabular}


Diferenciación Social en Patrones de Consumo de Exposiciones de arte, teatro,

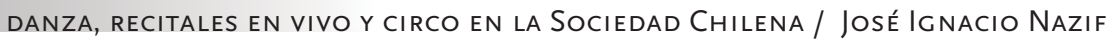

iii) Variables independientes

Las variables independientes que aquí se utilizan están orientadas a revisar cada una de las propuestas teóricas aquí revisadas.

a) Clase ocupacional: para revisar los postulados propuestos por Bourdieu, una alternativa de análisis es introducir la variable clase ocupacional. La construcción de dicha variable se hace a partir del trabajo realizado por Torche (2006). Dicha autora clasifica inicialmente los sujetos en 30 categorías ocupacionales, y posteriormente a partir de procedimientos teóricos y metodológicos, propone una nueva clasificación para la sociedad chilena en la cual hay nueve clases: Profesionales, Empleador, No Manual Calificado, No Manual No Calificado, Supervisores Manuales, Manuales Calificados, Manuales No Calificados y Trabajadores Agrícolas. Para determinar la clasificación ocupacional de cada persona en conformidad a este esquema se utilizaron las preguntas “¿Qué ocupación o tipo de trabajo desempeña usted actualmente?” y "En su trabajo usted es: (1) Empleador o patrón; (2) Trabajador por cuenta propia, independiente; (3) Asalariado, sector público; (4) Asalariado, sector privado; (5) Personal de servicio doméstico, puertas adentro; (6) Personal de servicio doméstico, puertas afuera ;(7) Familiar no remunerado; (8) Jubilado; (9) Dueña de casa".

b) Nivel educacional: para contrastar los postulados por Bourdieu, es necesario introducir la variable nivel educacional. Con dicha variable se podrá ver si la clasificación ocupacional predice el consumo cultural sin perder su carácter explicativo. Dicha variable fue operacionalizada de la siguiente manera: (1) 'Hasta nivel básico' (incluye las personas que nunca asistieron a ningún tipo de educación, kindergarten y básica); (2) 'Hasta Media o Humanidades' (incluye las personas que completaron su educación media o de humanidades como también aquellas que la terminaron); (3) 'Hasta Educación Superior Técnica' (incluye a las personas que realizaron cursos o los terminaron en los niveles correspondientes a Normal, Centros de Formación Técnica e Institutos profesionales). Finalmente la variable de referencia fue (0) 'Educación Universitaria' (se incluye todas las personas con cursos de pregrado y/o postgrados universitarios).

c) Edad:Dicha variable incluye la edad de los entrevistados, y sirve para identificar parte de la teoría cuasiposmoderna.

d) Género:Esta es una segunda variable para revisar la propuesta cuasiposmoderna. 'Mujer' fue codificado en (0) y 'Hombre' en (1).

e) Índice Realización individual de actividades artísticas: dicha variable es la 
última correspondiente a la propuesta de la versión cuasiposmoderna. Para determinar la anterior se consideró si en los últimos meses la persona había: (1) Sacado fotografias, elaborado videos o alguna otra creación audiovisual; (2) Hecho algún trabajo manual con fines artísticos o creativos (ej.: costura, cerámica, artesanía, etc.); (3) Tocado un instrumento, cantado o compuesto música; (4) Diseñado, dibujado, pintado o realizado una escultura; (5) Escrito cuentos, poesías o literatura en general; (6) Bailado o participado en una coreografia o espectáculo de expresión corporal; (7) Participado de una representación teatral. Los puntajes posibles estaban en una escala de 0 a $7 . \mathrm{El}$ alfa de Cronbach de este índice es de 0,68.

f) Participación de la familia: dicha variable es para revisar la propuesta clasificada como el eslabón perdido. Esta se constituye a partir de la pregunta: "Su padre, madre, tíos o abuelos, ¿realizan o realizaron alguna actividad artística?". Las respuestas negativas fueron codificadas como (0), mientras que las positivas como (1).

TABLA 2. Estadísticas DESCRIPTIVAS DE LAS VARIABLES INDEPENDIENTES

\begin{tabular}{|l|l|c|c|c|c|c|l|}
\hline \multicolumn{3}{|c|}{ Muestra 1 } & \multicolumn{3}{c|}{ Muestra 2 } \\
\hline $\begin{array}{l}\text { Variables } \\
\text { Independientes }\end{array}$ & Alternativa & $\%$ & Media & $\begin{array}{l}\text { Desviación } \\
\text { Standard }\end{array}$ & $\%$ & Media & $\begin{array}{l}\text { Desviación } \\
\text { Standard }\end{array}$ \\
\hline & Profesionales & 12,2 & & & 12,9 & & \\
\cline { 2 - 8 } & Empleador & 5,6 & & & 6,2 & & \\
\cline { 2 - 8 } & $\begin{array}{l}\text { No Manual } \\
\text { Calificado }\end{array}$ & 17,2 & & & 15,0 & & \\
\cline { 2 - 8 } & $\begin{array}{l}\text { No Manual } \\
\text { No Calificado }\end{array}$ & 7,6 & & & 7,6 & & \\
\cline { 2 - 8 } ocupacional & Independientes & 18,1 & & & 20,7 & & \\
\cline { 2 - 8 } & $\begin{array}{l}\text { Supervisores } \\
\text { Manuales }\end{array}$ & 7,6 & & & 8,4 & & \\
\cline { 2 - 8 } & $\begin{array}{l}\text { Manuales } \\
\text { Calificados }\end{array}$ & 16,8 & & & 15,6 & & \\
\cline { 2 - 8 } & $\begin{array}{l}\text { Manuales } \\
\text { No Calificados }\end{array}$ & 11,8 & & & 10,1 & & \\
\cline { 2 - 8 } & $\begin{array}{l}\text { Trabajadores } \\
\text { Agrícolas }\end{array}$ & 3,1 & & & 3,6 & & \\
\hline
\end{tabular}


Diferenciación Social en Patrones de Consumo de Exposiciones de arte, teatro, danza, ReCitAles en Vivo y CiRCo en la Sociedad Chilena / José IGnacio Nazif

\begin{tabular}{|c|c|c|c|c|c|c|c|}
\hline \multirow{4}{*}{$\begin{array}{l}\text { Nivel } \\
\text { educacional }\end{array}$} & $\begin{array}{l}\text { Educación } \\
\text { Universitaria }\end{array}$ & 22,1 & & & 23,6 & & \\
\hline & $\begin{array}{l}\text { Hasta Nivel } \\
\text { Básico }\end{array}$ & 19,7 & & & 19,3 & & \\
\hline & $\begin{array}{l}\text { Hasta Media } 0 \\
\text { Humanidades }\end{array}$ & 48,5 & & & 47,6 & & \\
\hline & $\begin{array}{l}\text { Hasta } \\
\text { Educación } \\
\text { Superior } \\
\text { Técnica }\end{array}$ & 9,7 & & & 9,5 & & \\
\hline Edad & & & 41,3 & 9,52 & & 41,5 & 9,54 \\
\hline \multirow{2}{*}{ Género } & (0) Mujer & 43,7 & & & 42,4 & & \\
\hline & (1) Hombre & 56,3 & & & 57,6 & & \\
\hline $\begin{array}{l}\text { Índice } \\
\text { realización } \\
\text { individual de } \\
\text { actividades } \\
\text { artísticas }\end{array}$ & & & 0,91 & 0,17 & & 0,91 & 0,17 \\
\hline \multirow{3}{*}{$\begin{array}{l}\text { Participación de } \\
\text { la familia }\end{array}$} & (0) No & 21,6 & & & 23,4 & & \\
\hline & (1) Sí & 78,4 & & & 76,6 & & \\
\hline & & \multicolumn{3}{|c|}{$\mathrm{N}=1853$} & \multicolumn{3}{|c|}{$\mathrm{N}=1349$} \\
\hline
\end{tabular}

\section{Resultados y análisis}

Para entender cómo está socialmente estratificado el consumo cultural se analizó cada una de las tres dimensiones. Para la dimensión (a) Asistencia a exposiciones de arte, se utilizó regresión logística dicotómica, mientras que regresión lineal para las dimensiones (b) Índice consumo de teatro, danza y recitales en vivo, y (c) Asistencia al circo.

Al analizar el consumo de exposiciones de arte (tabla 3) se puede apreciar a partir del modelo 1 que el análisis propuesto por Bourdieu en una primera instancia es acertado, ya que la categoría profesional es significativamente distinta a cada una de las categorías de la clasificación ocupacional introducidas. Por ejemplo al tomar sólo en consideración el valor correspondiente a la categoría manuales calificados $\left(\mathrm{e}^{7.156}\right)$, se puede señalar que una persona de ocupación profesional tiene 1282 veces más chances de asistir a una exposición de arte que una persona clasificada como manual calificado. Por otra parte, al revisar los exponenciales de los de cada una de las categorías de las ocupaciones desde empleador hasta trabajador agrícola se observa un aumento sostenido de este dato, ello implicaría que habría un consumo menor de 
exposiciones de arte a medida que se avanza desde la primera hasta la última categoría. Considerando que las exposiciones de arte serían una expresión cultural clasificada como highbrow, la distinción la tendría el grupo de personas profesionales puesto que ellos son los que consumirían esta forma de arte.

No obstante lo anterior, al introducir las categorías correspondientes de la variable educación (modelo 2) se observa que los postulados de Bourdieu comienzan a desvanecerse, ya que por un lado la condición profesional de los sujetos sólo sirve para distinguir la asistencia a exposiciones de arte con las ocupaciones que van desde no manuales no calificados hasta trabajadores agrícolas, y por otra parte ésta categoría deja de ser relevante para diferenciarlos de aquellos que son empleadores y no manuales calificados, dejando de ser ésta un conducta que distingue a la "elite". La diferenciación social en torno a este bien cultural comienza a trasladarse hacia la calidad educacional que la personas tienen (status), ya que a medida que los sujetos aumentan su nivel educacional mayores son las chances de asistir a exposiciones de arte. Con relación a algunos de los datos obtenidos, se puede señalar que los profesionales tienen 102 veces más chances de asistir a exposiciones de arte que aquellas personas ubicadas en la categoría manuales calificados $\left(\mathrm{e}^{4.635}\right)$, es decir, una vez introducida la variable nivel educacional, ésta produce un efecto directo en la disminución de las chances que un profesional tiene de consumir el bien analizado. Con respecto a la variable educacional se puede señalar que aquellas personas con educación universitaria tiene 684,71 más veces de ir a una exposición de arte que aquellas que asistieron hasta nivel básico $\left(\mathrm{e}^{6.529}\right)$. Es importante aclarar que si bien existe una alta correlación entre clase ocupacional y nivel educacional, realizar esta distinción analítica entre ambas variables reporta un resultado sobresaliente ya que el campo simbólico referido al consumo cultural tiene un mejor correlato sociológico con la segunda variable, es decir, la distribución del consumo de exposiciones de arte se observa con mayor precisión con las categorías educacionales que con las categorías ocupacionales.

$\mathrm{Al}$ analizar la propuesta correspondiente a la interpretación cuasiposmoderna (modelo 3), los resultados indican que ni edad ni género son adecuados para entender el consumo de este bien cultural. Sin embargo, la única variable que tiene un sentido predictor importante bajo esta mirada teórica es el índice realización individual de actividades artísticas. Por otra parte el signo negativo de este parámetro indica que entre más actividades artísticas hayan sido realizadas por la persona, mayores posibilidades de asistir a una exposición de arte ${ }^{8}$. Esto implicaría que para el consumo de este tipo de bien cultural habría un acentuado proceso de individualización que sobrepasaría sólo la subcultura estamental asociada a la clase social del sujeto. En palabras de Giddens, la constitución del self tendría una alta relevancia para entender esta elección ya que a medida que el sujeto experimenta directamente con otras actividades artísticas, el sujeto está en condiciones de asistir a exposiciones de arte. $8 \quad$ El obtenido para esta variable fue $(-3,474)$. Cabe recordar además que la asistencia a exposiciones de arte fue codificado como ' 0 ', mientras que la no asistencia como ' 1 '. 
Diferenciación Social en Patrones de Consumo de Exposiciones de arte, teatro, danza, ReCitales en Vivo y CiRco en la Sociedad Chilena / José IGnacio Nazif

Tomando a su vez las categorías de las variables clasificación ocupacional y educación en forma conjunta, se puede señalar en primer lugar que no hay un solapamiento entre estratificación social y estratificación cultural en los términos que propone Bourdieu, ya que la asistencia a exposiciones de arte sólo sirve para diferenciar a profesionales de supervisores manuales, manuales calificados, manuales no calificados y trabajadores agrícolas, pero no para distinguirlos de los empleadores, no manuales calificados, no manuales no calificados, e independientes. En segundo término, educación mantiene el carácter explicativo en su plenitud ya que aquellos que pasaron por la universidad tienen diferencias estadísticamente significativas con aquellas personas cuyos niveles educacionales son menores. Esto último implica que la noción weberiana de status, en contraste al concepto de clase, mantiene su sentido teórico para entender cómo está constituido el estilo de vida de las personas.

Tabla 3. Modelos de Regresión logística dicotómica para dimensión Asistencia a exposiciones de arte

\begin{tabular}{|c|c|c|c|c|c|}
\hline & & Modelo 1 & Modelo 2 & Modelo 3 & Modelo 4 \\
\hline \multirow{8}{*}{$\begin{array}{l}\text { Clase } \\
\text { ocupacional }\end{array}$} & Empleador & $\begin{array}{r}1,623^{*} \\
(, 239)\end{array}$ & $\begin{array}{l}1,087 \\
(, 252)\end{array}$ & $\begin{array}{l}1,005 \\
(, 267)\end{array}$ & $\begin{array}{l}1,011 \\
(, 271)\end{array}$ \\
\hline & $\begin{array}{l}\text { No Manual } \\
\text { Calificado }\end{array}$ & $\begin{array}{r}2,441^{* * *} \\
(, 179)\end{array}$ & $\begin{array}{l}1,434 \\
(, 198)\end{array}$ & $\begin{array}{l}1,226 \\
(, 211)\end{array}$ & $\begin{array}{l}1,241 \\
(, 213)\end{array}$ \\
\hline & $\begin{array}{l}\text { No Manual } \\
\text { No Calificado }\end{array}$ & $\begin{array}{r}3,748^{* * *+1+\dagger} \\
(, 236)\end{array}$ & $\begin{array}{r}1,696^{*} \\
(, 264)\end{array}$ & $\begin{array}{l}1,340 \\
(, 278)\end{array}$ & $\begin{array}{l}1,317 \\
(, 282)\end{array}$ \\
\hline & Independientes & $\begin{array}{r}3,960^{* * *}+t \dagger \\
(, 185)\end{array}$ & $\begin{array}{r}1,551^{*} \\
(, 219)\end{array}$ & $\begin{array}{l}1,374 \\
(, 234)\end{array}$ & $\begin{array}{l}1,379 \\
(, 236)\end{array}$ \\
\hline & $\begin{array}{l}\text { Supervisores } \\
\text { Manuales }\end{array}$ & $\begin{array}{r}7,013^{* * *+1+\dagger} \\
(, 272)\end{array}$ & $\begin{array}{r}2,831^{* * *} \\
(, 296)\end{array}$ & $\begin{array}{r}2,416^{* *} \\
(, 315)\end{array}$ & $\begin{array}{r}2,339^{*} \\
(, 316)\end{array}$ \\
\hline & $\begin{array}{l}\text { Manuales } \\
\text { Calificados }\end{array}$ & $\begin{array}{r}7,157^{* * *+t+\dagger} \\
(, 272)\end{array}$ & $\begin{array}{r}2,824^{* * *} \\
(, 240)\end{array}$ & $\begin{array}{r}2,289^{* *} \\
(, 258)\end{array}$ & $\begin{array}{r}2,276^{*} \\
(, 261)\end{array}$ \\
\hline & $\begin{array}{l}\text { Manuales } \\
\text { No Calificados }\end{array}$ & $\begin{array}{r}13,751^{* * * *+\dagger} \\
(, 280)\end{array}$ & $\begin{array}{r}4,635^{* * *} \\
(, 310)\end{array}$ & $\begin{array}{r}3,046^{* *} \\
(, 320)\end{array}$ & $\begin{array}{r}2,947^{*} \\
(, 323)\end{array}$ \\
\hline & $\begin{array}{l}\text { Trabajadores } \\
\text { Agrícolas }\end{array}$ & $\begin{array}{r}9,017^{* * * *+十 \dagger} \\
(, 425)\end{array}$ & $\begin{array}{r}3,263^{* * *} \\
(, 447)\end{array}$ & $\begin{array}{r}3,300^{*} \\
(, 475)\end{array}$ & $\begin{array}{r}3,225^{*} \\
(, 480)\end{array}$ \\
\hline \multirow{3}{*}{$\begin{array}{l}\text { Nivel } \\
\text { educacional }\end{array}$} & $\begin{array}{l}\text { Hasta Educación } \\
\text { Superior Técnica }\end{array}$ & & $\begin{array}{r}1,861^{* * *} \\
(, 240)\end{array}$ & $\begin{array}{r}1,654^{*} \\
(, 250)\end{array}$ & $\begin{array}{r}1,648^{*} \\
(, 252)\end{array}$ \\
\hline & $\begin{array}{l}\text { Hasta Media } 0 \\
\text { Humanidades }\end{array}$ & & $\begin{array}{r}2,831^{* * *} \\
(, 159)\end{array}$ & $\begin{array}{r}2,661^{* * *} \\
(, 167)\end{array}$ & $\begin{array}{r}2,548^{* * *} \\
(, 170)\end{array}$ \\
\hline & Hasta Nivel Básico & & $\begin{array}{r}6,529^{* * *} \\
(, 199)\end{array}$ & $\begin{array}{r}5,012^{* * *} \\
(, 209)\end{array}$ & $\begin{array}{r}4,561^{* * *} \\
(, 212)\end{array}$ \\
\hline
\end{tabular}


ReVista de Sociolocía 21 / 2007 ChILe Hoy

Facultad de Ciencias Sociales - Universidad de Chile

\begin{tabular}{|c|c|c|c|c|}
\hline Edad & & & $\begin{array}{l}1,000 \\
(, 007)\end{array}$ & $\begin{array}{l}1,000 \\
(, 347)\end{array}$ \\
\hline Género & & & $\begin{array}{l}1,232 \\
(, 130)\end{array}$ & $\begin{array}{l}1,293 \\
(, 131)\end{array}$ \\
\hline $\begin{array}{l}\text { Índice } \\
\text { realización } \\
\text { individual de } \\
\text { actividades } \\
\text { artísticas }\end{array}$ & & & $\begin{array}{r}, 023 * * * \\
(, 342)\end{array}$ & $\begin{array}{r}, 031^{* * *} \\
(, 007)\end{array}$ \\
\hline $\begin{array}{l}\text { Participación } \\
\text { de la familia }\end{array}$ & & & & $\begin{array}{r}1,997^{* * *} \\
(, 138)\end{array}$ \\
\hline Constante & $\begin{array}{l}, 808 \\
(, 134)\end{array}$ & $\begin{array}{l}, 700^{*} \\
(, 138)\end{array}$ & $\begin{array}{l}-, 084 \\
(, 007)\end{array}$ & $\begin{array}{r}, 300^{* *} \\
(, 461)\end{array}$ \\
\hline $\mathbf{N}$ & 1852 & 1852 & 1852 & 1852 \\
\hline Log likelihood & $1.932,41$ & $1.857,36$ & $1.716,26$ & $1.691,64$ \\
\hline
\end{tabular}

Coeficientes exp (B) y errores estándar entre paréntesis. Niveles de significación:

$* \mathbf{p}<0.05 / * * \mathrm{p}<0.01 / * * * \mathrm{p}<0.001$. Niveles de significación cuando la categoría de referencia es empleador: $\uparrow \mathrm{p}<0.05 /+\uparrow \mathrm{p}<0.01 /+\uparrow+\mathrm{p}<0.001$.

Con relación a la influencia de la familia (modelo 4) se puede apreciar que si un pariente cercano, sea este padre, madre, tíos o abuelos realiza o ha realizado una actividad artística, éste tiene una influencia positiva y significativa en la elección que un sujeto hace respecto de asistir a exposiciones de arte. Lo anterior implica que el proceso de socialización primaria de los sujetos en la sociedad influye fuertemente en el consumo de este tipo de expresión cultural. Esto es, la familia estaría efectivamente transmitiendo gustos artísticos a sus miembros menores o al menos incidiendo en el tipo de arte que los sujetos consumen. Se puede sugerir como mecanismo de transmisión la invitación que estos familiares hacen a sus descendientes a las distintas actividades artísticas que ellos mismos realizan. Al igual que el modelo 3, tanto la influencia del interés individual, el nivel educacional, y algunas categorías de la variable de clasificación ocupacional, contribuyen a que el sujeto opte por visitar las exposiciones de arte. 
Diferenciación Social en Patrones de Consumo de Exposiciones de arte, teatro, dANZA, ReCitAles en Vivo y CiRCo en la Sociedad Chilena / José IGnacio Nazif

Tabla 4. Modelos de Regresión para dimensión Índice consumo de TEATRO, DANZA Y RECITALES EN VIVO

\begin{tabular}{|c|c|c|c|c|c|}
\hline & & Modelo 5 & Modelo 6 & Modelo 7 & Modelo 8 \\
\hline \multirow{8}{*}{ Clase ocupacional $^{4}$} & Empleador & $\begin{array}{r}-, 104 \\
(, 086) \\
\end{array}$ & $\begin{array}{r}, 058 \\
(, 086) \\
\end{array}$ & $\begin{array}{r}, 113 \\
(, 083) \\
\end{array}$ & $\begin{array}{r}, 108 \\
(, 082) \\
\end{array}$ \\
\hline & $\begin{array}{l}\text { No Manual } \\
\text { Calificado }\end{array}$ & $\begin{array}{r}-, 240^{* * * *} \\
(, 063) \\
\end{array}$ & $\begin{array}{l}-, 023 \\
(, 067) \\
\end{array}$ & $\begin{array}{r}, 040 \\
(, 065) \\
\end{array}$ & $\begin{array}{r}, 035 \\
(, 065)\end{array}$ \\
\hline & $\begin{array}{l}\text { No Manual } \\
\text { No Calificado }\end{array}$ & $\begin{array}{r}-, 426^{* * *}+\dagger \\
(, 078)\end{array}$ & $\begin{array}{l}-, 095 \\
(, 084)\end{array}$ & $\begin{array}{l}-, 006 \\
(, 082)\end{array}$ & $\begin{array}{l}-, 002 \\
(, 081)\end{array}$ \\
\hline & Independientes & $\begin{array}{r}-, 542^{* * *}+\dagger \dagger \\
(, 062)\end{array}$ & $\begin{array}{r}-, 152^{* *} \\
(, 072)\end{array}$ & $\begin{array}{l}-, 081 \\
(, 069)\end{array}$ & $\begin{array}{l}-, 082 \\
(, 069)\end{array}$ \\
\hline & $\begin{array}{l}\text { Supervisores } \\
\text { Manuales }\end{array}$ & $\begin{array}{r}-, 634^{* * *}+\dagger \dagger \\
(, 078)\end{array}$ & $\begin{array}{r}-, 240^{* * *} \\
(, 085)\end{array}$ & $\begin{array}{l}-, 147 \\
(, 083)\end{array}$ & $\begin{array}{l}-, 139 \\
(, 083)\end{array}$ \\
\hline & $\begin{array}{l}\text { Manuales } \\
\text { Calificados }\end{array}$ & $\begin{array}{r}-, 627^{* * * *}+\dagger \dagger \\
(, 063) \\
\end{array}$ & $\begin{array}{r}-, 230^{* * *} \\
(, 073) \\
\end{array}$ & $\begin{array}{l}-, 124 \\
(, 073) \\
\end{array}$ & $\begin{array}{l}-, 123 \\
(, 072)\end{array}$ \\
\hline & $\begin{array}{l}\text { Manuales } \\
\text { No Calificados }\end{array}$ & $\begin{array}{r}-, 787^{* * *}+\dagger \dagger \\
(, 069)\end{array}$ & $\begin{array}{r}-, 333^{* * *} \\
(, 080)\end{array}$ & $\begin{array}{r}-, 197^{* *} \\
(, 077)\end{array}$ & $\begin{array}{r}-, 189^{* *} \\
(, 077)\end{array}$ \\
\hline & $\begin{array}{l}\text { Trabajadores } \\
\text { Agrícolas }\end{array}$ & $\begin{array}{r}-, 514^{* * * *}+\dagger \dagger \\
(, 107) \\
\end{array}$ & $\begin{array}{r}-, 081^{* *} \\
(, 111) \\
\end{array}$ & $\begin{array}{r}-, 011 \\
(, 107) \\
\end{array}$ & $\begin{array}{l}-, 006 \\
(, 107) \\
\end{array}$ \\
\hline \multirow{3}{*}{ Nivel educacional $^{5}$} & Hasta Nivel Básico & & $\begin{array}{r}-, 675^{* * *} \\
(, 062) \\
\end{array}$ & $\begin{array}{r}-, 560^{* * *} \\
(, 061) \\
\end{array}$ & $\begin{array}{r}-, 533^{* * * *} \\
(, 061) \\
\end{array}$ \\
\hline & $\begin{array}{l}\text { Hasta Media } 0 \\
\text { Humanidades }\end{array}$ & & $\begin{array}{r}-, 427^{* * *} \\
(, 052)\end{array}$ & $\begin{array}{r}-, 375^{* * *} \\
(, 050) \\
\end{array}$ & $\begin{array}{r}-, 357^{* * * *} \\
(, 050)\end{array}$ \\
\hline & $\begin{array}{l}\text { Hasta Educación } \\
\text { Superior Técnica }\end{array}$ & & $\begin{array}{r}-, 169^{* *} \\
(, 067)\end{array}$ & $\begin{array}{l}-, 119 \\
(, 064)\end{array}$ & $\begin{array}{l}-, 114 \\
(, 064)\end{array}$ \\
\hline Edad & & & & $\begin{array}{l}-, 002 \\
(, 002)\end{array}$ & $\begin{array}{l}-, 002 \\
(, 002)\end{array}$ \\
\hline Género & & & & $\begin{array}{r}-, 014 \\
(, 034)\end{array}$ & $\begin{array}{l}-, 026 \\
(, 034)\end{array}$ \\
\hline $\begin{array}{l}\text { Índice realización } \\
\text { individual de } \\
\text { actividades } \\
\text { artísticas }\end{array}$ & & & & $\begin{array}{r}1,120^{* * *} \\
(, 093)\end{array}$ & $\begin{array}{r}1,018^{* * *} \\
(, 095)\end{array}$ \\
\hline $\begin{array}{l}\text { Participación de la } \\
\text { familia }\end{array}$ & & & & & $\begin{array}{r}, 201^{* * *} \\
(, 040) \\
\end{array}$ \\
\hline Constante & & $\begin{array}{r}2,646^{* * *} \\
(, 048) \\
\end{array}$ & $\begin{array}{r}2,697^{* * *} \\
(, 048) \\
\end{array}$ & $\begin{array}{r}2,551^{* * *} \\
(, 103) \\
\end{array}$ & $\begin{array}{r}2,925^{* * * *} \\
(, 126) \\
\end{array}$ \\
\hline $\mathbf{N}$ & & 1853 & 1853 & 1853 & 1853 \\
\hline $\mathbf{R}^{2}$ & & .10 & .16 & .22 & .23 \\
\hline
\end{tabular}

Coeficientes (B) y errores estándar entre paréntesis. Niveles de significación: * p $<0.05 / * * p<$ $0.01 / * * * \mathrm{p}<0.001$. Niveles de significación cuando la categoría de referencia es empleador: $\dagger \mathrm{p}$ $<0.05 /$ † $\mathrm{p}<0.01 /+\uparrow \dagger \mathrm{p}<0.001$. 
Los resultados correspondiente a la dimensión índice consumo de teatro, danza y recitales en vivo (tabla 4) tienen implicancias muy parecidas a los resultados obtenidos para la asistencia a exposiciones de arte. Al ser sólo introducidas las categorías de la variable clasificación ocupacional (modelo 5), se aprecia que el grupo profesional y empleador tienden a obtener puntajes significativamente más altos que los otros grupos. Esto significa que dichos grupos cuando son comparados con los otros, asisten en mayor medida a presenciar obras teatrales y de danza, y recitales en vivo. Tomando en consideración tanto los resultados de los modelos 1 y 5 , y en términos de la distinción omnívoros-unívoros, se podría señalar que el grupo profesional manifiesta mayores características de omnívoros en comparación a todos los otros grupos de la clasificación ocupacional, ya que obtiene significativamente mayores puntajes en las dos dimensiones analizadas. En segundo término estaría el grupo empleadores, puesto que presenta diferencias estadísticas con los otros grupos (a excepción de los no manuales calificados para los resultados correspondientes al índice). Con respecto a lo planteado por Bourdieu, y tomando en conjunto el modelo 1 y 5 , se puede establecer que la presencia de un grupo omnívoro provoca un distanciamiento con los postulados de este autor ya que no habría claramente un grupo socioeconómico que monopoliza el consumo de los bienes culturales hasta ahora analizados. A lo sumo, y utilizando las categorías de este autor, se puede reconocer la existencia de dos grupos distinguidos, siendo el profesional el más distinguido, y el empleador el segundo. Pero precisamente esta ampliación en el consumo de estos bienes indica que no hay un solapamiento, a la Bourdieu, entre estratificación cultural y estratificación social.

Al introducir las categorías de la variable educación (modelo 6), se puede observar que el efecto en las categorías de la clasificación ocupacional es mucho mayor que cuando se analiza el consumo de exposiciones de arte (modelo 2), ya que el grupo profesional se distingue sólo de las ocupaciones que van desde los independientes hasta los trabajadores agrícolas, o en otras palabras el carácter distinguido lo tienen los profesionales, los empleadores, los no manuales calificados y los no manuales no calificados. Cuando son analizados los resultados correspondientes a las categorías de la variable educación, se puede apreciar que aquellas personas que tienen educación universitaria, tienden a tener signficativamente mayores puntajes que aquellos con niveles de educación inferior. Lo anterior signfica que la que distinción analítica propuesta por Weber, entre clases y status, es procedente, ya que la referencia simbólica se corresponde con mayor precisión con la estructura educacional de las personas, mientras que la estructura ocupacional es más difusa para estos términos.

La introducción de las variables correspondientes a la versión cuasiposmoderna (modelo 7) revela por un lado, dos efectos diferenciados para las variables clasificación y nivel educacional respectivamente, y por el otro un efecto positivo del índice de realización artística desarrollado. En primer lugar, la condición ocupacional de las personas deja prácticamente en su totalidad de ser relevante para entender cómo 
Diferenciación Social en Patrones de Consumo de Exposiciones de arte, teatro,

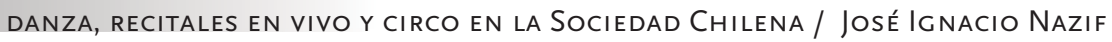

se configura el consumo de teatro, danza y recitales en vivo, ello porque el grupo profesional sólo se distingue de aquellos calificados como manuales no calificado. Esto último es muy relevante, con respecto a lo señalado por Bourdieu, ya que con ello queda establecido que las características asociadas a la estructura de clase son irrelevantes para entender el consumo de los bienes culturales analizados?

En segundo lugar, la variable educacional sigue vigente, pero en menor medida, ya que la educación universitaria es relevante para distinguir el consumo de los bienes analizados de aquellas personas con niveles educacionales de hasta educación básica y hasta educación media, pero no así con aquellos que declaran haber tenido educación superior técnica. Esto último puede estar relacionado con el hecho de que en dicho grupo están aquellas personas cuya educación fue normalista, es decir, profesores encargados de la formación de educación primaria de un número importante de personas. Particularmente con respecto a la distinción weberiana, cabe señalar que si bien ésta pierde cierta relevancia ante la introducción de las variables cuasiposmodernas, sigue siendo importante porque al ver los resultados de los parámetros se aprecia que el consumo de los bienes analizados es decreciente a medida que disminuye el nivel educacional de las personas.

Al analizar específicamente los resultados asociados a las variables cuasiposomodernas se puede apreciar que tanto género como edad no son significativos para entender las variaciones del índice de consumo cultural construido. Esto quiere decir que ser hombre o mujer, joven o adulto, no contribuye a visualizar diferenciaciones respecto del consumo de los bienes culturales en cuestión. Al analizar sin embargo el índice realización individual de actividades artísticas se puede observar que éste sí está relacionado positivamente con la asistencia al teatro, danza y a recitales de música en vivo. Al igual que en el modelo 3, la vocación artística de la persona tiene una repercusión positiva en esta dimensión. En consecuencia, en primera instancia se podría señalar que la extensión de la educación vivida en la sociedad chilena, tiene en sí un efecto positivo en la elección que hacen las personas puesto que éstas logran desarticular el peso de las herencias de clase. Sin embargo, al tomar los resultados de las categorías de la variable educación, se puede señalar que la búsqueda artística individual se enmarca necesariamente en la extensión del proceso educacional que tuvo el sujeto, y no en una búsqueda vocacional aislada de lo aprendido en el colegio y la universidad.

Finalmente ante la introducción de la variable asociada a la participación de la familia

\footnotetext{
Una interpretación ad-hoc para "salvar" los postulados de este autor sería ubicar el análisis exclusivamente en el ámbito subjetivo de los actores. En dicha instancia la elite podría seguir arguyendo tener el monopolio del canon, ya que aun cuando dicho grupo tenga objetivamente patrones de consumo similares a los otros grupos ocupacionales, las interpretaciones que ellos hacen de las obras en cuestión pueden ser enmarcadas en criterios artísticos 'superiores', puesto que ellos sí logran entender el verdadero sentido de las obras. Por ejemplo, todos pueden asistir a ver Antígona, pero sólo los miembros de la clase ocupacional "profesional" podrían entender el sentido que ocupa el coro en la obra.
} 
Revista de Sociología 21 / 2007 Chile Hoy

Facultad de Ciencias Sociales - Universidad de Chile

(modelo 8), los datos, por un lado, ratifican el análisis correspondiente al modelo 7, ya que, en primer lugar, la influencia de la ocupación disminuye fuertemente, luego el nivel educacional mantiene cierta prevalencia, y finalmente la variable Índice realización individual de actividades artísticas influye directa y positivamente. Por otra lado, estos resultados también indican que tener parientes ligados al desarrollo de actividades artísticas tiene un efecto positivo en un mayor consumo del índice consumo de teatro, danza y recitales en vivo. En consecuencia, tal como señala Tironi, Valenzuela y Scully, la familia ocupa un rol preponderante en la socialización de los chilenos, ya que las preferencias artísticas de éstos están asociadas a las experiencias que los parientes mayores tuvieron. En síntesis la formación de biografias personales ligadas al consumo de ciertos bienes culturales pasa necesariamente por aquello que los miembros mayores de las familias transmiten a sus herederos.

\section{Tabla 5. Modelos de Regresión para dimensión Asistencia al Circo}

\begin{tabular}{|c|c|c|}
\hline & & Modelo 9 \\
\hline \multirow{8}{*}{ Clase ocupacional $^{6}$} & Profesionales &,$- 057(, 094)$ \\
\hline & Empleador &,$- 008(, 101)$ \\
\hline & No Manual Calificado &,$- 053(, 081)$ \\
\hline & No Manual No Calificado &,$- 031(, 091)$ \\
\hline & Independientes &,$- 117(, 073)$ \\
\hline & Supervisores Manuales &,$- 016(, 090)$ \\
\hline & Manuales Calificados &, $028(, 079)$ \\
\hline & Trabajadores Agrícolas &,$- 071(, 116)$ \\
\hline \multirow{3}{*}{ Nivel educacional $^{7}$} & Hasta Media o Humanidades &,$- 011(, 053)$ \\
\hline & Hasta Educación Superior Técnica &,$- 013(, 081)$ \\
\hline & Universitaria y post-universitarias &,$- 122(, 072)$ \\
\hline Edad & &,$- 005^{* *}(, 002)$ \\
\hline Género & &, $015(, 041)$ \\
\hline $\begin{array}{l}\text { Índice realización individual de } \\
\text { actividades artísticas }\end{array}$ & &, $325^{* * *}(, 112)$ \\
\hline Participación de la familia & &, $076(046)$ \\
\hline Constante & & $1,711^{* * *}(, 165)$ \\
\hline $\mathbf{N}$ & & 1349 \\
\hline $\mathbf{R}^{2}$ & & .02 \\
\hline
\end{tabular}

Coeficientes (B) y errores estándar entre paréntesis. Niveles de significación: * p $<0.05$ / ** $<$ $0.01 / * * * \mathrm{p}<0.001$.

Los resultados correspondientes a la Dimensión Asistencia al circo (modelo 9) permiten analizar el consumo de este bien cultural en forma aislada, y también con relación a los otros bienes culturales previamente revisados. Primero, se puede apreciar 
Diferenciación Social en Patrones de Consumo de Exposiciones de arte, teatro, dANZA, RECitAles En Vivo y CiRCo en la Sociedad Chilena / José IGnacio Nazif

que las variables edad e Índice realización individual de actividades artísticas tienen una incidencia directa en una mayor asistencia al circo. Con relación a la primera variable se puede apreciar a partir del signo negativo de ese parámetro que entre más joven la persona mayor es la tendencia de asistir a espectáculos circenses. Con respecto a la segunda variable se observa que a medida que la persona realiza un número mayor de diversas actividades artísticas ésta se correlaciona positivamente con la cantidad de asistencia al $\operatorname{circo}^{10}$. Lo anterior implica que la perspectiva cuasiposmoderna, en comparación a las otras propuestas teóricas es más precisa para comprender la asistencia al circo, ya que es la única que presenta significancia estadística. En otras palabras, las categorías asociadas a clase, educación y familia aportan muy poco al entendimiento sobre el consumo de este bien cultural.

El resultado asociado a la edad de los individuos es muy llamativo, ya que este tipo de expresión tiene una tradición centenaria en este país, es decir, se hubiese esperado una mayor asistencia de los grupos de edades mayores a este tipo de espectáculos. En coherencia a lo anterior, una explicación posible es que las personas que van al circo -espectáculo dirigido principalmente al público infantil, lo hacen porque acompañan a los menores que habitan sus hogares ${ }^{11}$. Con relación al Índice realización individual de actividades artísticas se puede señalar, al igual que lo analizado en los otros bienes culturales, que la vocación artística de la persona permite explorar expresiones culturales de toda índole, siendo el caso del espectáculo circense una posibilidad más de búsqueda. En consecuencia la construcción de la biografia personal juega un rol muy importante en el plano de las elecciones de consumo artístico, sin embargo, cabe señalar que dicho proceso puede estar relacionado con la vocación del sujeto por convertirse en artista, más que por ser mero espectador de expresiones culturales.

Teniendo en consideración el consumo de todos los bienes culturales aquí analizados, cabe señalar que sólo una parte de la operacionalización de la proposición teórica cuasiposmoderna explicaría en mayor profundidad este fenómeno, ya que aquellas personas con experiencias personales directas en la creación de bienes culturales audiovisuales, esculturales, musicales literarios, de expresión corporal, o teatrales, son aquellos que en mayor medida asisten a exposiciones de arte, obras teatrales, espectáculos de danza, recitales en vivo y espectáculos circenses. Este mismo grupo a su vez, sería aquel considerado como el grupo omnívoro "duro", ya que su consumo atraviesa transversalmente ámbitos artísticos reconocidos tradicionalmente como highbrow y se extiende a un ámbito de orden popular como es el espectáculo circense.

\footnotetext{
A diferencia de los otros análisis, para el caso de esta dimensión sólo se insertó los resultados correspondientes a un único modelo, ya que cuando se siguió la metodología anterior, no se encontraron relaciones estadísticas significantes cada vez que se iban introduciendo las variables correspondientes a las propuestas teóricas.

Si efectivamente las personas que asisten al circo lo hacen debido a que acompañan a los infantes a estas actividades, una hipótesis asociada al consumo de este espectáculo por parte del grupo infantil sería que el imaginario infantil sintonizaría mejor con obras que resaltan lo mágico y extraordinario en instancias concretas (i.e. la cabeza del domador dentro de las fauces del león) versus aquellas constituidas por contenidos más abstractos. Agradezco a Pablo Morris por introducir la observación anterior.
} 
Sin embargo, hay que tener cierta cautela con este resultado porque como se aprecia en la tabla 2 el puntaje promedio es de 0,91 puntos, es decir, la gran mayoría de las personas entrevistadas no ha tenido experiencias directas en la creación de bienes artísticos como los mencionados. En otras palabras el grupo omnívoro por excelencia sería un subconjunto cuantitativamente menor en la sociedad chilena.

\section{Conclusión}

Los resultados de este trabajo sugieren que la estratificación social de los bienes culturales para la sociedad chilena varía según el tipo de expresión artística que se analiza. Ello implica que no hay una única respuesta teórica para entender el fenómeno en cuestión. En coherencia a lo anterior, es posible identificar dos focos interpretativos: primero, el consumo de algunos bienes está provocado por los procesos de modernización que esta sociedad tiene, puesto que éste encuentra su explicación en rasgos modernos y conservadores. Segundo, la aparición de un grupo omnívoro "duro" está caracterizada por elementos que sobrepasan las estructuras sociales tradicionales y modernas, hallando su referencia principal en instancias biográficas propias de los elementos cuasiposmodernos aquí descritos, sin embargo la aparición de este grupo hay que tomarla con cautela, debido a que es en términos cuantitativos de baja representación.

Con respecto a los procesos de modernización se puede señalar que la perdida de relevancia de la clase ocupacional, a medida que son introducidos otros factores en el consumo de los bienes culturales analizados (nivel educacional, familia artista, índice de realización de actividades individuales) es parte de un rasgo específico de la modernidad tardía de la sociedad chilena. En palabras de Larraín esto se ha producido dado que "la sociedad civil (esfera privada de los individuos, clases y organizaciones regidas por la ley civil) es débil, insuficientemente desarrollada y muy dependiente de los dictados del Estado y la política" (Larraín, 1997:328), en particular esto repercute en elites sin autonomía para desarrollar patrones de cultura distintivos. Con lo anterior se puede señalar, para el contexto chileno, que los postulados planteados por Bourdieu son débiles para entender el consumo de estos bienes culturales, pues en este plano la elite ocupacional no logra distinguirse de las otras capas sociales. Sin embargo al analizar los datos asociados a la introducción de la variable educacional surge una segunda hipótesis asociada a los procesos de modernización que Chile ha vivido. En este caso la ampliación de la cobertura del sistema educacional ha significado que las personas hayan adquirido nuevos códigos y conocimientos, los cuales les han permitido a los sujetos explorar un mayor número de expresiones culturales. En consecuencia, la distinción analítica propuesta por Weber, entre status y clase, sigue teniendo mucha relevancia, ya que esta primera categoría permite observar con mayor precisión diferencias culturales en la sociedad chilena. No obstante lo anterior, estos resultados no implican que la sociedad chilena haya eliminado el vínculo entre 
Diferenciación Social en Patrones de Consumo de Exposiciones de arte, teatro,

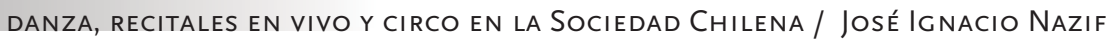

clase y status, por ejemplo Nuñez y Gutiérrez demuestran que en el mercado laboral aún persisten diferencias vinculadas al origen más que al mérito (Nuñez y Gutiérrez, 2004), sino más bien que el status es más apropiado que las categoría ocupacionales para entender ciertas distribuciones de consumo ocurridas en el campo cultural.

Dentro del proceso anteriormente descrito, la influencia que ejerce la familia en el consumo cultural puede también ser considerado un rasgo de una modernidad tardía. La presencia de un familiar (padre, madre, tíos o abuelos) vinculado a alguna actividad artística, tiene un efecto directo y positivo en el individuo. La familia, en consecuencia mantiene un fuerte carácter socializador, y la activación de algunos procesos de la sociedad chilena que facilitarían una institucionalización de la individualización no estarían rompiendo esta influencia. Como se sugirió más arriba un mecanismo social que contribuiría a explicar el efecto positivo de esta variable sería la imitación racional que los sujetos hacen de sus respectivos familiares, puesto que estos últimos tendrían un aura legitimizadora.

Los resultados asociados a la introducción del espectáculo circense sugieren la aparición de un grupo omnívoro duro, ello porque aquellas personas que obtienen puntajes altos en el Índice de realización individual de actividades artísticas, son aquellos que consumen en mayor medida cada uno de los bienes culturales aquí analizados. En tal sentido, estos patrones culturales, en conformidad a lo sugerido por Giddens y Beck respectivamente estarían siendo explicados por la institucionalización de la individualización, ya que son experiencias asociadas directamente a la autorrealización personal, superando en consecuencia estructuras sociales tradicionales y modernas. No obstante lo anterior, la cautela que hay que tener con dichos resultados es el número de personas que tienen dichas características, ya que como se señalo más arriba el puntaje promedio de este índice es muy bajo $(0,91)$ en una escala de 0 a 7 . Esto en consecuencia significa que si bien la existencia de un grupo omnívoro está presente en la sociedad chilena, su baja representación hablaría exclusivamente de escasos procesos individuales vinculados a vocaciones ocupacionales de orden artístico.

Para terminar, cabe señalar que este trabajo sólo abre preguntas respecto a cómo el proceso de modernización pautea las conductas culturales declaradas de la sociedad chilena, ya que si bien se hace evidente la tensión entre estructuras tradicionales y modernas para identificar parte del consumo de los bienes culturales aquí analizados, existe un gran repertorio de bienes que ha sido dejado de lado (i.e. música, literatura, programas televisivos, cine, etc.). El estudio de estos bienes puede posteriormente inclinar la balanza hacia algunas de las estructuras conservadoras o modernas, y en consecuencia profundizar respecto a cómo la sociedad chilena tiene estructurado el consumo cultural. Por otra parte, la construcción del discurso en torno al consumo cultural de los sujetos en este trabajo no ha sido incorporada, y esto significa que elementos interpretativos respecto al contenido de los bienes culturales han sido 
ReVista de Sociolocía $21 / 2007$ CHILE Hoy

Facultad de Ciencias Sociales - Universidad de Chile

excluidos. Para lo anterior es necesario llevar a cabo estudios cualitativos sobre estas áreas, ya que de esa manera se generaría conocimiento sobre la dimensión subjetiva que conforma la identidad cultural de los grupos sociales. Esto último es de suma relevancia porque con dicho conocimiento se puede establecer además una tipificación del consumo cultural en torno a diferentes cánones.

\section{Bibliografía}

Barozet, E.: "El Valor Histórico del Pituto" Revista de Sociología 20, 2006, 69-96. Facultad de Ciencias Sociales Universidad de Chile.

Bellavance, G, M.Valex et M Ratté:"Le Goût des Autres: Une analyse des Répertoires Culturels de Nouvelles Elites Omnivore” Sociologie et Sociétés, XXXVI, 2004, 27-57.

Biggs, S. et al.: "Baby Boomers and Adult Ageing in Public Policy: The Changing Relationship between Production and Consumption"

http://www.consume.bbk.ac.uk/working_papers/Biggsetal2006.doc

: "New Consumers? The Social and Cultural Significance of Children's Fashion Consumption"

http://www.consume.bbk.ac.uk/working_papers/Boden\%20Working\%20Paper2. doc

Bourdieu, P.: La Distinction: Critique Sociale du Jugement. Editions de Minuit, 1979.

: "Condition de Classe et Position de Classe". En Archives Europénnes

de Sociologie,VII, 1966.

Brunner, J.J. y G. Elacqua: "Informe Capital Humano en Chile" Universidad Adolfo Ibáñez. Escuela de Gobierno, Mayo 2003 (159 p).

Bryson, B.: "Anything but Heavy Metal: Symbolic Exclusion and Musical Dislikes." American Sociological Review, 61(5), 1996, 884-899.

Chan, T. W. and J. Goldthorpe: "Social Stratification and Cultural Consumption: Music in England" European Sociological Review, 2007(a), 23(1), 1-19.

:"Social Status and Newspaper Readership". American Journal of Sociology, 112, 2007(b), 1095-1134.

:"Class and Status: The Conceptual Distinction and its Empirical Relevance" Sociology working papers 2006-03, Department of Sociology, University of Oxford. 
Diferenciación Social en Patrones de Consumo de Exposiciones de arte, teatro,

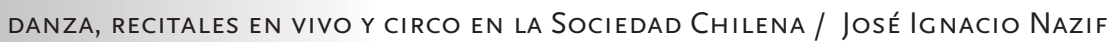

:"The Social Stratification of Theatre, Dance and Cinema

Attendance". Cultural Trends Vol. 14(3), No. 55, September 2005, 193-212.

: "Social Stratification and Cultural Consumption:The Visual Arts in

England" Poetics (forthcoming, 2007).

Contreras, D. et al.: "Dinámica de la Pobreza y Movilidad Social: Chile 1996-2001" Universidad de Chile. Departamento de Economía, Agosto 2004 (29 p).

Elster, J.: Sour Grapes: Studies in the Subversion of Rationality. Cambridge: Cambridge University Press, 1983

: "Snobs (Review of Pierre Bourdieu (1979), La Distinction)". En London Review of Books, November 5, 1981, vol. 3, no 20, 10-12.

Fernández, C:"La Ruptura de la Exclusividad del Gusto a través de la Música Sinfónica como Espacio de Integración Social para Niños y Jóvenes de Sectores Populares: Las Orquestas Juveniles e Infantiles de Chile"

http://www.cybertesis.cl/tesis/uchile/2006/fernandez_c/html/index-frames.html

Fontaine, A.: Equidad y Calidad en la Educación: Cinco Proposiciones Interrelacionadas ; Revista Estudios Públicos, № 87, 2002, 5-38.

García-Albarido,A.: Música y Habitus .

http://www.cybertesis.cl/tesis/uchile/2005/garcia-albarido_a/html/index-frames. html

Gans, H. J. : Popular Culture and High Culture: An Analysis and Evaluation of Taste. Basic Books, New York, revised edition. 1999.

Giddens, A.: Modernity and Self-identity: Self and Society in the Late Modern Age. Polity, Cambridge 1991.

Hedström, P.: "Rational Imitation". En P. Hedström and R. Swedberg (eds.) Social Mechanisms: Analytical Approach to Social Theory (Studies in Rationality \& Social Change). Cambridge University Press, 1998.

Higgs. P. et al.: "From passive to active consumers? Trends in ownership of key goods in retired and non-retired households in the UK from 1968-2001"

http://www.consume.bbk.ac.uk/working_papers/HiggsetalJune2006.doc

Jelin, E.: "Las Familias en América Latina". En Regina Rodríguez (ed.) Familias Siglo XXI. ISIS Ediciones de las mujeres, Santiago 1994. 
Revista de Sociología 21 / 2007 Chile Hoy

Facultad de Ciencias Sociales - Universidad de Chile

Larraín, J.: La Identidad Chilena. Santiago. LOM, 2001

: "La Trayectoria Latinoamericana A La Modernidad" Revista

Estudios Públicos, N 66, 1997,313-333.

Larrañaga, O.: “ Participación Laboral de la Mujer, 1958-2003”. En J.S. Valenzuela, E. Tironi y T. R. Scully (eds.) El Eslabón Perdido. Familia. Modernización y Bienestar en Chile. Taurus, 2006.

Lipovetsky, G. et E. Roux: Le Luxe Éternel: De l'âge du Sacré au Temps des Marques. France, 2003, Gallimard.

Livingston, S.: "Children online: Consumers or Citizens". Cultures of Consumption Working Papers Series. 2004. Www.consume.bbk.ac.uk/working_papers/ Livingstoneworkingpaper.doc

Martens, L., D. Southerton, and S. Scott: "Bringing Children (and Parents) into the Sociology of Consumption:Towards a Theoretical and Empirical Agenda" En Journal of Consumer Culture, July 1, 2004; 4(2): 155 - 182.

Mukerji, Ch. and M. Schudson: Rethinking Popular Culture: Contemporary Perspectives on Cultural Studies. Los Angeles. University of California Press, 1991.

Núñez, J. y Gutiérrez R.: "Class Discrimination and Meritocracy in the Labor Market: Evidence from Chile". En Estudios de Economía.Vol. 31 - No 2, Diciembre 2004, 113-132.

Peterson, R.A. and R. M. Kern: "Changing Highbrow Taste: From Snob to Omnivore.” En American Sociological Review, vol. 61, No. 5, October 1996, 900-907.

Peterson, R.A. and A.Simkus: "How musical tastes mark occupational status groups". En M. Lamont and M. Fournier, (eds), Cultivating Differences: Symbolic Boundaries and the Making of Inequality, chapter Seven, pages 152-186. 1992. University of Chicago Press, Chicago.

Savage, M. et al.: "Cultural Capital in the UK: a Preliminary Report Using Correspondence Analysis" CRESC Working Paper Series. Working Paper No. 4. Center for Research on Socio-Cultural Change (CRESC) Faculty of Social Sciences, The Open University.

Southerton, D.: "Boundaries of 'Us' and 'Them': Class, Mobility and Identification in a New Town Southerton”. En Sociology 2002; vol. 36, No. 1, 171-193.

Tironi, E., J. S.Valenzuela y T. R. Schully: “Familia en Chile los Impactos de la Modernización”. En J.S.Valenzuela, E. Tironi y T. R. Scully (eds) El Eslabón Perdido. Familia. 
Diferenciación Social en Patrones de Consumo de Exposiciones de arte, teatro, danZA, ReCitales en Vivo y CIRCo en la Sociedad Chilena / José IGnacio Nazif

Modernización y Bienestar en Chile. Taurus, 2006.

Torche, F::"Una Clasificación de Clases para la Sociedad Chilena" Revista de Sociología 20, 2006, 15-43. Facultad de Ciencias Sociales. Universidad de Chile.

: "Unequal but Fluid: Social Mobility in Chile in Comparative Perspective". American Sociological Review, 70(3), 2005, 422-450.

Valenzuela, E. y M.S. Herrera: “Tiempo, Trabajo y Familia”. En J.S.Valenzuela, E. Tironi y T. R. Scully (eds) El Eslabón Perdido. Familia. Modernización y Bienestar en Chile. Taurus, 2006.

Valenzuela, E.: "Padres Involucrados y Uso de Drogas: un Análisis Empírico”. Revista Estudios Públicos, № 101, 2005, 147-164.

van den Berg, A.: "Is Sociological Theory Too Grand for Social Mechanisms". En P. Hedstrom and R. Swedberg (eds.) Social Mechanisms: Analytical Approach to Social Theory (Studies in Rationality \& Social Change). Cambridge University Press, 1998.

van Eijck, K.: "Social Differentiation in Musical Taste Patterns" Social Forces, vol. 79, No. 3, March 2001, 1163-1185.

: "The Impact of Family Participation and Educational Attainment on Cultural Consumption: A Sibling Analysis” Poetics, vol. 25, 1997, 195-224.

Winch, R. F: The Modern Family, New York: Henry Holt and Co., 1963. 